\title{
Passive Imaging of Moving Targets Using Distributed Apertures in Multiple-Scattering Environments
}

\author{
Ling Wang ${ }^{a}$ and Birsen Yazıc1 ${ }^{b}$ \\ ${ }^{a}$ Department of Information and Communication Engineering, Nanjing University of \\ Aeronautics and Astronautics, Nanjing, Jiangsu 210016 China; \\ ${ }^{b}$ Department of Electrical, Computer and System Engineering, Rensselaer Polytechnic \\ Institute, Troy, NY 12180 USA
}

\begin{abstract}
We present a novel passive image formation method for moving targets using distributed apertures capable of exploiting information about multiple-scattering in the environment. We assume that the environment is illuminated by non-cooperative transmitters of opportunity with unknown location and unknown transmitted waveforms. We develop a passive measurement model that relates the scattered field from moving targets at a given receiver to the scattered field at other receivers. We formulate the passive imaging problem as a generalized likelihood ratio test for a hypothetical target located at an unknown position, moving with an unknown velocity. We design a linear discriminant functional by maximizing the Signal-to-Noise Ratio (SNR) of the test-statistic, and use the resulting position- and velocity-resolved test-statistic to form the image. Our imaging method can determine the two- or three-dimensional velocity vector as well as the two- or three-dimensional position vector of a moving target without the knowledge of transmitter locations and transmitted waveforms. We present numerical experiments to demonstrate the performance of our passive imaging method operating in multiplescattering environments. The results show that the point spread function of the reconstructed images improves when the information about multiple scattering is exploited.
\end{abstract}

Keywords: Passive Radar, Imaging, Moving Targets, Distributed Apertures, Multiple-Scattering, Generalized Likelihood Ratio Test

\section{INTRODUCTION}

With the rapid growth of broadcasting stations, mobile phone base stations, communication and navigation satellites, as well as relatively low cost and rapid deployment of receivers, passive radar imaging using transmitters of opportunity has emerged as an active area of research in recent years.

A number of passive moving target detection and imaging approaches have been presented in the literature. ${ }^{1-18}$ We present a new passive image formation method for moving targets using a sparse array of receivers and non-cooperative transmitters of opportunity operating in multiple-scattering environments, to reconstruct the distribution of targets in position and velocity spaces. To the best of our knowledge, our method is the first in the literature that exploits multiple-scattering for passive imaging of moving targets.

We use the shoot-and-bounce model to model the wave propagation in multiple-scattering environments. We develop a new passive measurement model that relates the Doppler as well as the delay information measured at a receiver location to the delay and Doppler information measured at other receiver locations due to a hypothetical moving target in position and velocity spaces. We use the new passive measurement model to address the moving target imaging problem as a position- and velocity-resolved binary hypothesis testing, which has its root in Generalized Likelihood Ratio Test (GLRT). ${ }^{19,20}$ The test-statistic produced by the hypothesis testing can be viewed as a superposition of filtered, delayed and scaled (or dilated) correlations of measurements

Further author information: (Send correspondence to B.Y.)

B.Y.: E-mail: yazici@ecse.rpi.edu, Telephone: +1(518)276 2905, Fax: +1(518)276 6261

L.W.:E-mail: wanglrpi@gmail.com

Algorithms for Synthetic Aperture Radar Imagery XVIII, edited by Edmund G. Zelnio, Frederick D. Garber, Proc. of SPIE Vol. 8051, 80510U · C 2011 SPIE · CCC code: 0277-786X/11/\$18 · doi: 10.1117/12.883828 
at different receivers. We use the position- and velocity-resolved test-statistics to form an image in position and velocity spaces.

We present numerical simulations to demonstrate the performance of our passive moving target imaging algorithms. The results show the improvements in the point spread function of the reconstructed images when the information about multiple scattering is exploited, as well as potential artifacts.

While our treatment focuses primarily on radar imaging, our method is directly applicable to passive imaging of moving objects in seismic, acoustic and microwave imaging.

The rest our paper is organized as follows: In Section 2, we describe the models for a moving target, incident and scattered fields using the shoot-and-bounce model, and present the passive measurement model for a moving target in a multiple-scattering environment. In Section 3, we address the moving target imaging problem in position and phase spaces within the GLRT framework using the passive measurement model. In Section 4, we present numerical simulations. Section 5 concludes our discussion.

\section{PASSIVE MEASUREMENT MODEL FOR MOVING TARGETS}

We reserve $\mathbf{x}$ to denote location in 3D Euclidean space and $\boldsymbol{x}$ to denote location in 2D space. We denote operators $\left(\mathcal{P}, \mathcal{S}\right.$, etc.) with calligraphic letters. For a function $f, \hat{f}$ denotes its Fourier transform and $f^{*}$ denotes its complex conjugate. Bold font denotes vector quantities. Non-bold italic font denotes scalar quantities.

\subsection{Model for the scattered field}

The propagation of electromagnetic waves due to an arbitrary source distribution $s(\mathbf{x}, t)$ in a medium can be described using the scalar wave equation: ${ }^{21-23}$

$$
\left[\nabla^{2}-c^{-2}(\mathbf{x}, t) \partial_{t}^{2}\right] E(\mathbf{x}, t)=s(\mathbf{x}, t)
$$

where $c$ is the wave speed in the medium and $E$ is the electric field. The propagation medium is characterized by the Green's function satisfying

$$
\left[\nabla^{2}-c_{0}^{-2} \partial_{t}^{2}\right] g(\mathbf{x}, \mathbf{z}, t)=\delta(\mathbf{x}-\mathbf{z}) \delta(t)
$$

The wave speed $c$ can be expressed in terms of the background propagation speed, $c_{0}$ and the perturbation due to deviation from the background reflectivity. Let $q_{\mathbf{v}}$ denote the phase-space distribution, at time $t=0$, of scatterers moving with velocity $\mathbf{v}$. The moving scatterers in the spatial volume $d^{3} x$ (at $\mathbf{x}$ ) give rise to $c^{-2}(t, \mathbf{x})=c_{0}^{-2}+\int q_{\mathbf{v}}(\mathbf{x}-\mathbf{v} t) d \mathbf{v} \cdot{ }^{24}$

We assume that the electromagnetic waves decay rapidly as they penetrate the ground. ${ }^{25}$ We then write $q_{\mathbf{v}}(\mathbf{x}-\mathbf{v} t)$ in terms of the two-dimensional location and two-dimensional velocity as follows:

$$
q_{\mathbf{v}}(\mathbf{x}-\mathbf{v} t)=q_{\boldsymbol{v}}(\boldsymbol{x}-\boldsymbol{v} t) \delta\left(x_{3}-h(\boldsymbol{x})\right) \delta\left(v_{3}-D h(\boldsymbol{x}) \cdot \boldsymbol{v}\right)
$$

where $\mathbf{x}=\left(\boldsymbol{x}, x_{3}\right), \boldsymbol{x} \in \mathbb{R}^{2}$ and $\mathbf{v}=\left(\boldsymbol{v}, v_{3}\right), \boldsymbol{v} \in \mathbb{R}^{2}, h: \mathbb{R}^{2} \rightarrow \mathbb{R}$ represents the ground topography and $D h(\boldsymbol{x})=\left[\begin{array}{ll}\frac{\partial h}{\partial x_{1}} & \frac{\partial h}{\partial x_{2}}\end{array}\right]$.

Let $E^{\mathrm{sc}}$ denote the scattered field due to the moving perturbation $q_{\boldsymbol{v}}$. Then, under the Born approximation, ${ }^{26}$ the scattered field is modeled as

$$
E^{\mathrm{sc}}(\mathbf{x}, t)=\int g(\mathbf{x}, \boldsymbol{y}, t-\tau) q_{\boldsymbol{v}}(\boldsymbol{y}-\boldsymbol{v} \tau) \partial_{\tau}^{2} E^{\mathrm{in}}(\boldsymbol{y}, \tau) d \tau d \boldsymbol{y} d \boldsymbol{v}
$$

where we define $g(\mathbf{x}, \boldsymbol{y}, t)$ as the $3 \mathrm{D}$ Green's function of the background environment that is equal to $g(\mathbf{x},(\boldsymbol{y}, h(\boldsymbol{y})), t)$. $E^{\text {in }}$ denotes the incident field due to the source distribution $s(\mathbf{x}, t)$ modeled as

$$
E^{\text {in }}(\boldsymbol{y}, t)=\int g(\boldsymbol{y}, \mathbf{z}, t-\tau) s(\mathbf{z}, \tau) d \tau d \mathbf{z}
$$


where $g(\boldsymbol{y}, \mathbf{z}, t)$ is the 3D Green's function defined as $g((\boldsymbol{y}, h(\boldsymbol{y})), \mathbf{z}, t)$.

For multiple transmitters with isotropic antennas, say $M$, the source distribution can be expressed as

$$
s(\mathbf{x}, t)=\sum_{q=1}^{M} \delta\left(\mathbf{x}-\mathbf{z}_{q}\right) p_{q}\left(t+T_{\mathbf{z}_{q}}\right)
$$

where $\mathbf{z}_{q}, q=1, \cdot, M$ denotes the location of the $q^{\text {th }}$ transmitter transmitting the waveform $\hat{p}_{q}$ starting at time $t=T_{\mathbf{z}_{q}}$. Thus, (5) can be expressed as

$$
E^{\mathrm{in}}(\boldsymbol{y}, t)=\sum_{q=1}^{M} \int \underbrace{g\left(\boldsymbol{y}, \mathbf{z}_{q}, t-\tau\right) p_{q}\left(\tau+T_{\mathbf{z}_{q}}\right)}_{E_{q}^{\operatorname{in}}\left(\boldsymbol{y}, \mathbf{z}_{q}, t\right)} d \tau
$$

where $E_{q}^{\text {in }}$ represents the incident field on the target due to the $q^{\text {th }}$ transmitter.

Note that isotropic antenna assumption is not necessary for the rest of our development. The results in the following sections can be extended to incorporate realistic antenna models in a straightforward manner.

Performing the change of variables $\boldsymbol{y}^{\prime}=\boldsymbol{y}-\boldsymbol{v} \tau$, (4) becomes

$$
E^{\mathrm{sc}}(\mathbf{x}, t)=\int g\left(\mathbf{x}, \boldsymbol{y}^{\prime}+\boldsymbol{v} \tau, t-\tau\right) q_{\boldsymbol{v}}\left(\boldsymbol{y}^{\prime}\right) \partial_{\tau}^{2} E^{\mathrm{in}}\left(\boldsymbol{y}^{\prime}+\boldsymbol{v} \tau, \tau\right) d \tau d \boldsymbol{y}^{\prime} d \boldsymbol{v}
$$

where $E^{\text {in }}\left(\boldsymbol{y}^{\prime}+\boldsymbol{v}, \tau\right)$ is given in (7) with $\boldsymbol{y}$ replaced by $\boldsymbol{y}^{\prime}+\boldsymbol{v} \tau$.

\subsection{Measurement Model For Multiple-scattering Environments}

For multiple-scattering environments, we consider a multi-bounce, multiple-scattering environment model. ${ }^{27-29}$ We assume that there are $L$ point scatterers in the background medium and consider only the propagation paths originating from the target to a background scatterer or from the background scatterer to the receiver. This results in $L$ multi-path bounces between the target and background medium. We further assume that all multi-path bounces are specular reflections and therefore model the environment as a collection of mirror planes. In this case, the Green's function of the background can be approximated by the following shoot-and-bounce model: ${ }^{27}$

$$
\begin{aligned}
\hat{g}(\mathbf{x}, \mathbf{y}, \omega) & =\frac{\mathrm{e}^{-\mathrm{i} k|\mathbf{x}-\mathbf{y}|}}{4 \pi|\mathbf{x}-\mathbf{y}|}+\sum_{l=1}^{L} a_{l} \frac{\mathrm{e}^{-\mathrm{i} k\left|\mathbf{x}^{l}-\mathbf{y}\right|}}{4 \pi\left|\mathbf{x}^{l}-\mathbf{y}\right|} \\
& =\sum_{l=0}^{L} a_{l} \frac{\mathrm{e}^{-\mathrm{i} k\left|\mathbf{x}^{l}-\mathbf{y}\right|}}{4 \pi\left|\mathbf{x}^{l}-\mathbf{y}\right|}, a_{0}=1, \mathbf{x}^{0}=\mathbf{x}
\end{aligned}
$$

where $k=\omega / c_{0}, \mathbf{x}^{l}$ is reflection of $\mathbf{x}$ about the mirror plane, $\mathcal{A}_{l}$, with respect to the $l$ th,$(l=1, \cdots, L)$ multi-path bounce and $a_{l}$ are the corresponding attenuation coefficients. We define the receivers located at $\mathbf{x}^{l},(l=1, \cdots, L)$ as mirror receivers.

Note that (10) allows us to readily compare the Point Spread Function (PSF) of the imaging operator obtained for the free-space to the one obtained for the multiple-scattering environment. We can express (10) as

$$
\hat{g}(\mathbf{x}, \mathbf{y}, \omega)=\sum_{l=0}^{L} a_{l} \hat{g}_{0}\left(\mathbf{x}^{l}, \mathbf{y}, \omega\right) .
$$

where $g_{0}\left(\mathbf{x}^{l}, \mathbf{y}, \omega\right)$ denotes the free-space Green's function given by

$$
g_{0}\left(\mathbf{x}^{l}, \mathbf{y}, \omega\right)=\frac{\mathrm{e}^{-\mathrm{i} k\left|\mathbf{x}^{l}-\mathbf{y}\right|}}{4 \pi\left|\mathbf{x}^{l}-\mathbf{y}\right|} .
$$


Thus, substituting (11) into (8), under the slow-mover assumption, using the scattered field obtained for free-space case, ${ }^{30}$ in Fourier domain the measurement at the receiver located at $\mathbf{x}$ contaminated with additive thermal noise $n(t)$ can be modeled as

$$
\hat{m}(\omega)=\sum_{l=0}^{L} a_{l} \int \hat{g}_{0}\left(\mathbf{x}^{l}, \boldsymbol{y}, \omega\right) q_{\boldsymbol{v}}(\boldsymbol{y}) \mu_{\boldsymbol{y}, \boldsymbol{v}, \mathbf{x}^{l}}^{2} \omega^{2} \hat{\tilde{E}}^{\text {in }}\left(\boldsymbol{y}, \mu_{\boldsymbol{y}, \boldsymbol{v}, \mathbf{x}^{l}} \omega\right) d \boldsymbol{y} d \boldsymbol{v}+\hat{n}(\omega)
$$

where

$$
\hat{\tilde{E}}^{\text {in }}(\boldsymbol{y}, \omega)=-\sum_{q=1}^{M} \sum_{\nu=1}^{L} \frac{1}{\tilde{\mu}_{\boldsymbol{y}, \boldsymbol{v}, \mathbf{z}_{q}^{\nu}}^{2}} \hat{E}_{q}^{\text {in }}\left(\boldsymbol{y}, \mathbf{z}_{q}^{\nu}, \frac{\omega}{\tilde{\mu}_{\boldsymbol{y}, \boldsymbol{v}, \mathbf{z}_{q}^{\nu}}}\right)
$$

with $\mathbf{z}_{q}^{0}=\mathbf{z}_{q}$ and $\mathbf{z}_{q}^{\nu}, \nu=1, \cdots, L$ being the reflections of the transmitters located at $\mathbf{z}_{q}$ about the plane $\mathcal{A}_{\nu}$. We define these transmitters as mirror transmitters. We assume that $n(t)$ is zero-mean with finite second-order statistics.

(13) shows that the measurement at the receiver consists of the scattered signals due to direct-path propagation and different multi-path propagations. Since the multi-path components contain the target information, they can be potentially exploited to improve imaging performance.

$\hat{E}_{q}^{\text {in }}\left(\boldsymbol{y}, \mathbf{z}_{q}^{\nu}, \frac{\omega}{\tilde{\mu}_{\boldsymbol{y}, v, \mathbf{z}_{q}^{\nu}}}\right)$ in $(14)$ is given as follow:

$$
\hat{E}_{q}^{\mathrm{in}}\left(\boldsymbol{y}, \mathbf{z}_{q}^{\nu}, \frac{\omega}{\tilde{\mu}_{\boldsymbol{y}, \boldsymbol{v}, \mathbf{z}_{q}^{\nu}}}\right)=\frac{1}{\tilde{\mu}_{\boldsymbol{y}, \boldsymbol{v}, \mathbf{z}_{q}^{\nu}}} \hat{g}_{0}\left(\mathbf{y}, \mathbf{z}, \frac{\omega}{\tilde{\mu}_{\boldsymbol{y}, \boldsymbol{v}, \mathbf{z}_{q}^{\nu}}}\right) \hat{p}_{q}\left(\frac{\omega}{\tilde{\mu}_{\boldsymbol{y}, \boldsymbol{v}, \mathbf{z}_{q}^{\nu}}}\right) \mathrm{e}^{\mathrm{i} \frac{\omega}{\tilde{\mu}_{\boldsymbol{y}, \boldsymbol{v}, \mathbf{z}_{q}^{\nu}}} T_{\mathbf{z}_{q}^{\nu}}} .
$$

Note that since the waveforms $\hat{p}_{q}, q=1, \cdots, M$ are typically band-limited, we restrict our analysis to some range of $\omega$.

In (13) and (14), $\mu_{\boldsymbol{y}, \boldsymbol{v}, \mathbf{x}^{l}}$ is given by

$$
\mu_{\boldsymbol{y}, \boldsymbol{v}, \mathbf{x}^{l}}=1+\widehat{\mathbf{y}-\mathbf{x}^{l}} \cdot \mathbf{v} / c_{0}
$$

which denotes the Doppler-scale-factor observed at location $\mathbf{x}$ due to a moving target ${ }^{27}$ with velocity $\boldsymbol{v}$ at location $\boldsymbol{y}$ associated with the $l^{\text {th }}$ multi-path propagation. $\tilde{\mu}_{\boldsymbol{y}, \boldsymbol{v}, \mathbf{z}_{q}^{\nu}}$ is given by

$$
\tilde{\mu}_{\boldsymbol{y}, \boldsymbol{v}, \mathbf{z}_{q}^{\nu}}=1-\widehat{\mathbf{y}-\mathbf{z}_{q}^{\nu}} \cdot \mathbf{v} / c_{0},
$$

which denotes the Doppler-scale-factor observed by the moving target ${ }^{27}$ located at $\boldsymbol{y}$ moving with velocity $\boldsymbol{v}$ due to a waveform transmitted from $\mathbf{z}_{q}$ along the $\nu^{\text {th }}$ multi-path bounce.

\subsection{Passive measurement model}

In the analysis that follows, we consider a sparse distribution of $N$ receivers located at $\mathbf{x}_{i}, i=1, \cdots, N$ and $M$ transmitters located at $\mathbf{z}_{q}, i=1, \cdots, M$. The receivers and transmitters may be arbitrarily located with several hundred wavelengths apart with no assumption that transmitters and receivers are colocated (see Fig. 1). Non-identical waveforms may be transmitted from different transmitters.

For passive detection and imaging applications, the information about the transmitted waveforms and the location of the transmitters may not be available. Therefore, we develop an alternative passive measurement model that expresses scattered field at each receiver in terms of the scattered field at a different receiver. This model is based on the fact that the measurements at all receiver are due to the same incident field $\tilde{E}^{\text {in }}$, target velocity $\boldsymbol{v}$, and the phase space distribution, $q_{\boldsymbol{v}}(\boldsymbol{y})$. The model involves back propagating the measurement at a receiver location to a hypothetical target location moving with a hypothetical velocity and then forward propagating the resulting field to another receiver location.

We use (13) to define the forward-propagation operator, $\mathcal{P}_{\boldsymbol{y}, \boldsymbol{v}, i}$, with respect to the $i^{\text {th }}$ receiver as follows:

$$
\mathcal{P}_{\boldsymbol{y}, \boldsymbol{v}, i}[u](\omega)=\sum_{l=0}^{L} a_{l} \mathcal{G}_{\boldsymbol{y}, i}^{l} \mathcal{S}_{\boldsymbol{v}, i}^{l}[u](\omega)
$$




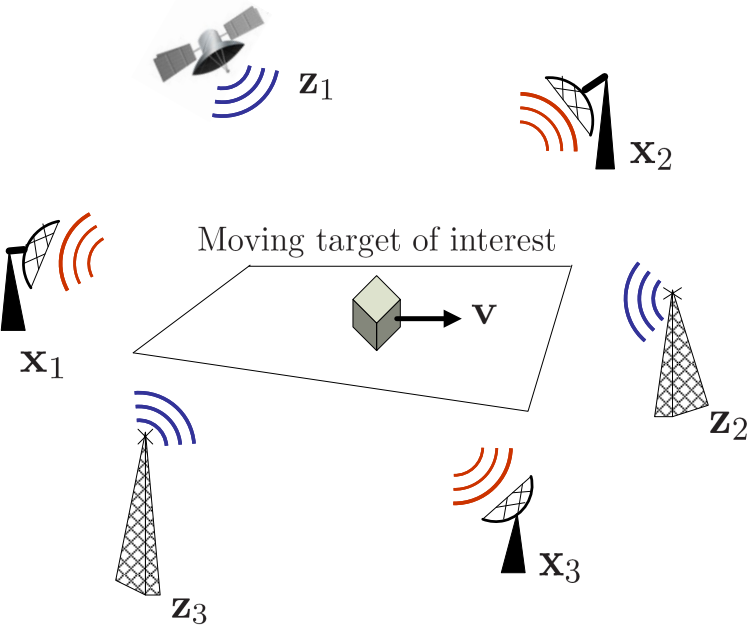

Figure 1: The figure illustrates a typical distributed aperture setup with receive and transmit antenna elements that are several hundred wavelengths apart. $\mathbf{x}_{i}, i=1, \cdots, 3$ denote receivers and $\mathbf{z}_{i}, i=1, \cdots, 3$ denote transmitters.

where

$$
u(\boldsymbol{y}, \boldsymbol{v}, \omega)=q_{\boldsymbol{v}}(\boldsymbol{y}) \omega^{2} \hat{\tilde{E}}^{\text {in }}(\boldsymbol{y}, \omega)
$$

$\mathcal{S}_{\boldsymbol{v}, i}^{l}$ is the scaling operator that accounts for the Doppler effect observed by the $i^{\text {in }}$ receiver due to a moving target with velocity $\boldsymbol{v}$ at location $\boldsymbol{y}$ associated with the $l^{\text {th }}, l=1, \cdots, L$ multi-path bounce; $\mathcal{G}_{\boldsymbol{y}, i}^{l}$ is the operator that accounts for the wave propagation in the stationary background from the target to the $i^{\text {th }}$ receiver via the $l^{\text {th }}, l=1, \cdots, L$ multi-path bounce; and $a_{l}$ is the corresponding attenuation coefficient defined as above. Note that in (19), $u$ is viewed as a function of $\boldsymbol{\omega}$ only, and $\boldsymbol{y}$ and $\boldsymbol{v}$ are fixed parameters.

We assume that the moving target is composed of a collection of point targets in position and velocity spaces. We define $\mathcal{S}_{\boldsymbol{v}, i}^{l} l=0, \cdots, L$ as

$$
\mathcal{S}_{\boldsymbol{v}, i}^{l}[u](\omega)=\mu_{\boldsymbol{y}, \boldsymbol{v}, \mathbf{x}_{i}^{l}} u\left(\boldsymbol{y}, \boldsymbol{v}, \mu_{\boldsymbol{y}, \boldsymbol{v}, \mathbf{x}_{i}^{l}} \omega\right)
$$

and $\mathcal{G}_{\boldsymbol{y}, i}^{l} l=0, \cdots, L$ as

$$
\mathcal{G}_{\boldsymbol{y}, i}^{l}[u](\omega)=\hat{g}_{0}\left(\mathbf{x}_{i}^{l}, \boldsymbol{y}, \omega\right) u(\boldsymbol{y}, \boldsymbol{v}, \omega)
$$

where $\hat{g}_{0}\left(\mathbf{x}_{i}^{l}, \boldsymbol{y}, \omega\right)$ is the Fourier transform of the 3D free-space Green's function $g_{0}\left(\mathbf{x}_{i}^{l},(\boldsymbol{y}, h(\boldsymbol{y})), t\right)$ given in $(12)$.

We define the back-propagation operator as the adjoint of $\mathcal{P}_{\boldsymbol{y}, \boldsymbol{v}, i}$ and denote it with $\mathcal{P}_{\boldsymbol{y}, \boldsymbol{v}, i}^{\dagger}$. Using (18), we express $\mathcal{P}_{\boldsymbol{y}, \boldsymbol{v}, i}^{\dagger}$ as

$$
\mathcal{P}_{\boldsymbol{y}, \boldsymbol{v}, i}^{\dagger}=\sum_{l=0}^{L} a_{l}^{*} \mathcal{S}_{\boldsymbol{v}, i}^{l, \dagger} \mathcal{G}_{\boldsymbol{y}, i}^{l, \dagger}
$$

where $\mathcal{S}_{\boldsymbol{v}, i}^{l, \dagger}$ and $\mathcal{G}_{\boldsymbol{y}, i}^{l, \dagger}$ are the adjoint of $\mathcal{S}_{\boldsymbol{v}, i}^{l}$ and $\mathcal{G}_{\boldsymbol{y}, i}^{l}$, respectively.

For a given measurement $\hat{m}(\omega), \mathcal{S}_{\boldsymbol{v}, i}^{l, \dagger}$ and $\mathcal{G}_{\boldsymbol{y}, i}^{l, \dagger}$ are given as follows:

$$
\mathcal{S}_{\boldsymbol{v}, i}^{l, \dagger}[\hat{m}](\omega)=\hat{m}\left(\frac{\omega}{\mu_{\boldsymbol{y}, \boldsymbol{v}, \mathbf{x}_{i}^{l}}}\right)
$$

and

$$
\mathcal{G}_{\boldsymbol{y}, i}^{l, \dagger}[\hat{m}](\omega)=\hat{g}^{*}\left(\mathbf{x}_{i}^{l}, \boldsymbol{y}, \omega\right) \hat{m}(\omega)
$$


Let $\hat{m}_{i}$ denote the measurement at the $i^{\text {th }}$ receiver. We can now express the measurement, $\hat{m}_{i}$, at the $i^{\text {th }}$ receiver in terms of the measurement, $\hat{m}_{j}$, at the $j^{\text {th }}$ receiver by back-propagating $\hat{m}_{j}$ measured at $\mathbf{x}_{j}$ to a hypothetical target location with a hypothetical velocity via the back-propagation operator; and then forward propagating the resulting field to $\mathbf{x}_{i}$ via the forward-propagation operator. Note that in the back-propagation operation, not only the noise-free measurement but also the noise at the $j^{\text {th }}$ receiver are back-propagated. Thus, we have

$$
\hat{m}_{i}(\omega)=\mathcal{P}_{\boldsymbol{y}, \boldsymbol{v}, i} \mathcal{P}_{\boldsymbol{y}, \boldsymbol{v}, j}^{\dagger} \hat{m}_{j}(\omega)+\hat{n}_{i}(\omega) .
$$

For a moving point target model given by

$$
q_{\boldsymbol{v}}(\boldsymbol{y})=\rho \delta\left(\boldsymbol{y}-\boldsymbol{y}_{0}\right) \delta\left(\boldsymbol{v}-\boldsymbol{v}_{0}\right)
$$

where $\rho$ is the reflectivity of the point target located at $\boldsymbol{y}_{0}$, at time $t=0$, moving with velocity $\boldsymbol{v}_{0}$, the forwardpropagating operator in (18) reduces to

$$
\mathcal{P}_{\boldsymbol{y}_{0}, \boldsymbol{v}_{0}, i}[u](\omega)=\sum_{l=0}^{L} a_{l} \hat{g}_{0}\left(\mathbf{x}_{i}^{l}, \boldsymbol{y}_{0}, \omega\right) \mu_{\boldsymbol{y}_{0}, \boldsymbol{v}_{0}, \mathbf{x}_{i}^{l}} \hat{u}\left(\mu_{\boldsymbol{y}_{0}, \boldsymbol{v}_{0}, \mathbf{x}_{i}^{l}}, \omega\right) .
$$

The back-propagation operator in (22) becomes

$$
\mathcal{P}_{\boldsymbol{y}_{0}, \boldsymbol{v}_{0}, i}^{\dagger}\left[\hat{m}_{i}\right](\omega)=\sum_{l=0}^{L} a_{l}^{*} \hat{m}_{i}\left(\frac{\omega}{\mu_{\boldsymbol{y}_{0}, \boldsymbol{v}_{0}, \mathbf{x}_{i}^{l}}}\right) \hat{g}_{0}^{*}\left(\mathbf{x}_{i}^{l}, \boldsymbol{y}_{0}, \frac{\omega}{\mu_{\boldsymbol{y}_{0}, \boldsymbol{v}_{0}, \mathbf{x}_{i}^{l}}}\right) .
$$

Using (27) and (28), for a moving point target, (25) becomes

$$
\hat{m}_{i}(\omega)=\sum_{l, l^{\prime}=0}^{L} a_{l} a_{l^{\prime}}^{*} \hat{g}_{0}\left(\mathbf{x}_{i}^{l}, \boldsymbol{y}_{0}, \omega\right) \hat{g}_{0}^{*}\left(\mathbf{x}_{j}^{l^{\prime}}, \boldsymbol{y}_{0}, \gamma_{\boldsymbol{y}_{0}, \boldsymbol{v}_{0}}^{i j, \beta} \omega\right) \mu_{\boldsymbol{y}_{0}, \boldsymbol{v}_{0}, \mathbf{x}_{i}^{l}} \hat{m}_{j}\left(\gamma_{\boldsymbol{y}_{0}, \boldsymbol{v}_{0}}^{i j, \beta} \omega\right)+\hat{n}_{i}(\omega)
$$

where $\gamma_{\boldsymbol{y}_{0}, \boldsymbol{v}_{0}}^{i j, \beta}, \beta=\left(l, l^{\prime}\right)$ is the ratio of the Doppler-scale-factors associated with the multi-path propagation between the target and the $i^{\text {th }}$ and $j^{\text {th }}$ receivers. It has the form

$$
\gamma_{\boldsymbol{y}_{0}, \boldsymbol{v}_{0}}^{i j, \beta}:=\frac{\mu_{\boldsymbol{y}_{0}, \boldsymbol{v}_{0}, \mathbf{x}_{i}^{l}}}{\mu_{\boldsymbol{y}_{0}, \boldsymbol{v}_{0}, \mathbf{x}_{j}^{l^{\prime}}}}=\frac{1+\widehat{\mathbf{y}_{0}-\mathbf{x}_{i}^{l}} \cdot \mathbf{v}_{0} / c_{0}}{1+\widehat{\mathbf{y}_{0}-\mathbf{x}_{j}^{l^{\prime}}} \cdot \mathbf{v}_{0} / c_{0}} \quad \beta=\left(l, l^{\prime}\right) .
$$

Note that $\mathbf{y}_{0}=\left(\boldsymbol{y}_{0}, h\left(\boldsymbol{y}_{0}\right)\right)$ and $\mathbf{v}_{0}=\left(\boldsymbol{v}_{0}, D h\left(\boldsymbol{y}_{0}\right) \cdot \boldsymbol{v}_{0}\right)$. We refer to $\gamma_{\boldsymbol{y}_{0}, \boldsymbol{v}_{0}}^{i j, \beta}, \beta=\left(l, l^{\prime}\right)$ as the passive-Doppler-scalefactor for the $i^{\text {th }}$ and $j^{\text {th }}$ receivers due to $l^{\text {th }}, l^{\text {th }}$ multi-path bounces.

Substituting the Fourier transform of the free-space Green's function into (29), we obtain

$$
\hat{m}_{i}(\omega)=\sum_{l, l^{\prime}=0}^{L} \frac{a_{l} a_{l^{\prime}}^{*} \mu_{\boldsymbol{y}_{0}, \boldsymbol{v}_{0}, \mathbf{x}_{i}^{l}}}{(4 \pi)^{2}\left|\mathbf{x}_{j}^{l^{\prime}}-\mathbf{y}_{0}\right|\left|\mathbf{x}_{i}^{l}-\mathbf{y}_{0}\right|} \mathrm{e}^{-\mathrm{i} k\left(\left|\mathbf{x}_{i}^{l}-\mathbf{y}_{0}\right|-\gamma_{\boldsymbol{y}_{0}, v_{0}}^{i j, \beta}\left|\mathbf{x}_{j}^{l^{\prime}}-\mathbf{y}_{0}\right|\right)} \hat{m}_{j}\left(\gamma_{\mathbf{y}_{0}, \boldsymbol{v}_{0}}^{i j, \beta} \omega\right)+\hat{n}_{i}(\omega)
$$

where $k=\omega / c_{0}$.

We next form a vector measurement model by taking one of the receivers as a reference. Without loss of generality, we take the $j^{\text {th }}$ receiver as a reference and form the following measurement vector:

$$
\mathbf{m}=\left[\begin{array}{llll}
\hat{m}_{1} & \hat{m}_{2} & \cdots & \hat{m}_{N}
\end{array}\right]^{T} .
$$

Similarly, we can vectorize the "reference measurements" and the noise as follows:

$$
\begin{aligned}
\mathbf{m}_{\mathbf{r}} & =\left[\begin{array}{llll}
\hat{m}_{j} & \hat{m}_{j} & \cdots & \hat{m}_{j}
\end{array}\right]^{T} \\
\mathbf{n} & =\left[\begin{array}{llll}
\hat{n}_{1} & \hat{n}_{2} & \cdots & \hat{n}_{N}
\end{array}\right]^{T}
\end{aligned}
$$


where $\hat{n}_{i}, i \neq j$ is the additive thermal noise at the $i^{\text {th }}$ receiver. Note that $\mathbf{m}, \mathbf{m}_{\mathbf{r}}$ and $\mathbf{n}$ are all $(N-1)$ dimensional vectors.

The composition of the back-propagation and forward-propagation operators can be represented as a diagonal matrix given by

$$
\mathbf{P}_{\boldsymbol{y}, \boldsymbol{v}}=\operatorname{diag}\left[\mathcal{P}_{\boldsymbol{y}, \boldsymbol{v}, 1} \mathcal{P}_{\boldsymbol{y}, \boldsymbol{v}, j}^{\dagger} \cdots \mathcal{P}_{\boldsymbol{y}, \boldsymbol{v}, N} \mathcal{P}_{\boldsymbol{y}, \boldsymbol{v}, j}^{\dagger}\right]
$$

where $i \neq j$ and $\mathbf{P}_{\boldsymbol{y}, \boldsymbol{v}}$ is $(N-1) \times(N-1)$.

Using (25), (32)-(35), we form a vectorized passive measurement model as follows:

$$
\mathbf{m}(\omega)=\mathbf{P}_{\boldsymbol{y}, \boldsymbol{v}} \mathbf{m}_{\mathbf{r}}(\omega)+\mathbf{n}(\omega)
$$

for some range of $\omega$. Note that in (36), all operations are understood to be elementwise.

\section{IMAGE FORMATION}

We use a hypothesis testing based approach to address the passive imaging problem which has its root in the Generalized Likelihood Ratio Test (GLRT) ${ }^{19,20}$ We set up a position- and velocity-resolved binary hypothesis testing and next determine a test-statistic for each location $(\boldsymbol{y}, h(\boldsymbol{y})) \in \mathbb{R}^{3}$ and each velocity $(\boldsymbol{v}, D h(\boldsymbol{y}) \cdot \boldsymbol{v}) \in \mathbb{R}^{3}$ in the position and velocity spaces using the passive measurement model for moving targets developed in subsection 2.3. The image is then formed in the $(\boldsymbol{y}, \boldsymbol{v})$ domain with the position- and velocity-resolved test-statistic.

We consider the following test of binary hypothesis for each location and velocity in $(\boldsymbol{y}, \boldsymbol{v})$ space:

$$
\begin{array}{ll}
\mathcal{H}_{0}: & \mathbf{m}=\mathbf{n} \\
\mathcal{H}_{1}: & \mathbf{m}=\mathbf{P}_{\boldsymbol{y}, \boldsymbol{v}} \mathbf{m}_{\mathbf{r}}+\mathbf{n}
\end{array}
$$

where $\mathbf{P}_{\boldsymbol{y}, \boldsymbol{v}}, \mathbf{m}_{\mathbf{r}}, \mathbf{m}$ and $\mathbf{n}$ are as defined in (32)-(36).

The null hypothesis states that the measurement is due to noise whereas the alternative hypothesis states that the measurement is due to a target located at $\boldsymbol{y}$ moving with velocity $\boldsymbol{v}$.

We design the following linear discriminant functional to address the binary hypothesis testing problem,

$$
\lambda=\langle\mathbf{m}, \mathbf{w}\rangle:=\int \mathbf{w}^{H} \mathbf{m} d \omega=\sum_{i, i \neq j} \int w_{i}^{*}(\omega) \hat{m}_{i}(\omega) d \omega
$$

where $\lambda$ denote the the output of the discriminant functional, which we call the test-statistic, and $\mathbf{w}$ is a template given by

$$
\mathbf{w}=\left[\begin{array}{llll}
w_{1} & w_{2} & \cdots & w_{N}
\end{array}\right]^{T} .
$$

We determine the test-statistic by maximizing the Signal-to-Noise Ratio (SNR) of $\lambda$. Under the assumption that the noise at different receivers are wide sense stationary and mutually uncorrelated, the resulting optimal linear template is given by

$$
\mathbf{w}_{\mathrm{opt}}=\overline{\mathbf{S}}^{-1} \mathbf{P}_{\boldsymbol{y}, v} \overline{\mathbf{m}}_{\mathbf{r}}
$$

where $\overline{\mathbf{S}}^{-1}$ is the inverse of $\overline{\mathbf{S}}$ defined by

$$
\overline{\mathbf{S}}(\omega)=\int 1 / 2\left(\mathbf{R}_{1}\left(\omega, \omega^{\prime}\right)+\mathbf{R}_{0}\left(\omega, \omega^{\prime}\right)\right) d \omega^{\prime}
$$

with $\mathbf{R}_{0}=\operatorname{Cov}\left[\mathbf{m} \mid \mathcal{H}_{0}\right]$ and $\mathbf{R}_{1}=\operatorname{Cov}\left[\mathbf{m} \mid \mathcal{H}_{1}\right]$ where Cov denotes the covariance operator. One can show that $\overline{\mathbf{S}}^{-1}$ can be approximated by a diagonal matrix. We denote diagonal elements of $\overline{\mathbf{S}}^{-1}$ by $\bar{S}_{i}^{-1}(\omega), i=1, \cdots, N$ and $i \neq j$, which is a function of the power spectral density function of noise at the $i^{\text {th }}$ receiver and the kernel of $\mathbf{P}_{\boldsymbol{y}, \boldsymbol{v}}$. 
Using the moving point target model given by (26), for a hypothetical point target located at $\boldsymbol{y}$, moving with a hypothetical velocity $\boldsymbol{v}$, each component of the optimal template becomes

$$
w_{i}=\sum_{l, l^{\prime}=0}^{L} \frac{a_{l} a_{l^{\prime}}^{*} \mu_{\boldsymbol{y}, \boldsymbol{v}, \mathbf{x}_{i}^{l}}}{(4 \pi)^{2}\left|\mathbf{x}_{j}^{l^{\prime}}-\mathbf{y}\right|\left|\mathbf{x}_{i}^{l}-\mathbf{y}\right|} \bar{S}_{i}^{-1}(\omega) \mathrm{e}^{-\mathrm{i} k\left(\left|\mathbf{x}_{i}^{l}-\mathbf{y}\right|-\gamma_{\boldsymbol{y}, \boldsymbol{v}}^{i j, \beta}\left|\mathbf{x}_{j}^{l^{\prime}}-\mathbf{y}\right|\right)} \mathrm{E}\left[\hat{m}_{j}\left(\gamma_{\boldsymbol{y}_{0}, \boldsymbol{v}_{0}}^{i j, \beta} \omega\right)\right]
$$

where $i=1, \cdots, N$ and $i \neq j$ and $\gamma_{\boldsymbol{y}, \boldsymbol{v}}^{i j, \beta}, \beta=\left(l, l^{\prime}\right)$ is given by (30).

In the summation in (42), the first term involves scaling due to geometrical spreading factors, the Dopplerscale-factor and the attenuation coefficients associated with the multi-path propagation. The second term is a prewhitening filter due to colored noise. The third term involves delay due to the path difference between the two receivers and a temporal dilation due to the passive-Doppler-scale-factor, and the last term involves averaged dilated reference measurement. Note that the path difference in the exponential term considers all the possible propagation paths between the $i^{\text {th }}$ and $j^{\text {th }}$ receivers, including the path difference between the direct-paths, multi-paths, and the direct-path back-propagation and $l^{\text {th }}(l=1, \cdots, L)$ multi-path forward propagation and vice versa between the two receivers.

Thus, the output of the linear discriminant functional can be viewed as a superposition of the correlations between the filtered, delayed, scaled (or dilated) replica of the reference measurement $m_{j}$ and the measurement $m_{i}, i \neq j$, which is given by

$$
\lambda(\boldsymbol{y}, \boldsymbol{v})=\sum_{i, i \neq j}^{N} \sum_{l, l^{\prime}=0}^{L} \int \frac{a_{l} a_{l^{\prime}}^{*} \mu_{\boldsymbol{y}, \boldsymbol{v}, \mathbf{x}_{i}^{l}}}{(4 \pi)^{2}\left|\mathbf{x}_{j}^{l^{\prime}}-\mathbf{y}\right|\left|\mathbf{x}_{i}^{l}-\mathbf{y}\right|} \mathrm{E}\left[m_{j}^{\prime}\left(\frac{t-\left|\mathbf{y}-\mathbf{x}_{i}^{l}\right| / c_{0}}{\gamma_{\boldsymbol{y}, \boldsymbol{v}}^{i j, \beta}}+\frac{\left|\mathbf{y}-\mathbf{x}_{j}^{l^{\prime}}\right|}{c_{0}}\right)\right] m_{i}^{*}(t) d t
$$

where $m_{j}^{\prime}(t)$ is the filtered version of $m_{j}(t)$ with the filtering given by $\bar{S}_{i}^{-1}(\omega)$. Note that for free-space, $l, l^{\prime}=0$ and $\mathbf{x}_{i}^{0}=\mathbf{x}_{i}, \mathbf{x}_{j}^{0}=\mathbf{x}_{j}$, the test-statistic in (43) reduces to the one in passive imaging of moving targets using sparse distributed apertures in free-space in ${ }^{30}$, up to scaling terms.

\section{NUMERICAL SIMULATIONS}

\subsection{Settings}

We conducted numerical simulations to verify the theory and to demonstrate the performance of our passive imaging method using a moving point target model.

We considered a scene of size $[0,3 \mathrm{e} 3] \times[0,3 \mathrm{e} 3] \mathrm{m}^{2}$ with flat topography. We discretized the scene into $201 \times 201$ pixels where $[0,0,0] \mathrm{m}$ and $[3 \mathrm{e} 3,3 \mathrm{e} 3,0] \mathrm{m}$ correspond to the pixels $(1,1)$ and $(201,201)$, respectively. We assumed that the target velocity is in the range of $[-20,20] \times[-20,20] \mathrm{m} / \mathrm{s}$. We discretized the velocity plane into $401 \times 401$ pixels where $[-20,-20,0] \mathrm{m}$ and $[20,20,0] \mathrm{m}$ correspond to the pixels $(1,1)$ and $(401,401)$, respectively.

The point target with unit reflectivity was assumed to be located at $\left[\begin{array}{lll}2.5 \mathrm{e} 3 & 2 \mathrm{e} 3 & 0\end{array}\right]^{T} \mathrm{~m}$ moving with velocity $[-10,15] \mathrm{m} / \mathrm{s}$.

We assumed that there were five receivers and a single transmitter present in the scene. The single transmitter was assumed to be located at $[1.5 \mathrm{e} 3,0,6]^{T} \mathrm{~m}$. The receivers were assumed to lie on the x-axis, equidistant form each other in the range of $[0,3 \mathrm{e} 3]^{T} \mathrm{~m}$. Both the transmitter and receivers were assumed to be on the same $z$-plane, $z=6$.

We considered a specular reflecting wall located at $x=0$. Thus, the multiple-scattering environment was modeled with the shoot-and-bounce model with one extra path. For ease of comparison, we presented the results for both free-space and multiple-scattering environments.

In all the experiments, the noise was simulated as an additive white Gaussian process.

Taking into account the waveforms of opportunity available in practice, we used transmitted waveforms which have high Doppler resolution and acceptable range resolution. Examples of such waveforms include wireless network (or WiFi) signals, Digital Video Broadcasting Terrestrial (DVB-T) signals and WiMAX ${ }^{31}$ waveforms which have relatively large bandwidth that offers reasonable range resolution. We refer to such 
waveforms as the high-Doppler and good-range resolution waveforms. We simulated a high-Doppler and goodrange resolution waveform as a continuous-wave $(\mathrm{CW})$ with $4 \mathrm{GHz}$ carrier frequency, $0.1 \mathrm{~s}$ duration, and with an additional frequency modulation, which results in a bandwidth of $7.5 \mathrm{MHz}$. Note that such a waveform provides about $19.5 \mathrm{~m}$ range resolution and $0.375 \mathrm{~m} / \mathrm{s}$ radial velocity resolution in monostatic operations.

We reconstructed the four-dimensional test-statistic image in $(\boldsymbol{y}, \boldsymbol{v})\left(\right.$ or $\left.\left(y_{1}, y_{2}, v_{1}, v_{2}\right)\right)$ coordinates. However, in order to facilitate visualization and performance evaluation, we generated three two-dimensional images from the original four dimensional image: The fist image, which we refer to as the peak-value image, was generated by choosing the maximum value of the four-dimensional image for each velocity $\left(v_{1}, v_{2}\right)$. We then, chose the maximum value, $\tilde{\boldsymbol{v}}$, of the peak-value image as the estimate of the velocity. The second image, which we refer to as the position-image, is the cross-section of the four-dimensional image, for $\boldsymbol{v}=\tilde{\boldsymbol{v}}$. We take the maximum value, $\tilde{\boldsymbol{y}}$, of the position-image as an estimate of the target's position. The third image, which we refer to as the velocity-image, is the cross-section of the four-dimensional image, for $\boldsymbol{y}=\tilde{\boldsymbol{y}}$.

\subsection{Results}

The reconstructed images in free-space using high-Doppler and good-range resolution waveform are shown in Fig. 2. The reconstructed images in multiple-scattering environments using the the same waveforms are shown in Fig. 3. We see that in both free-space and multi-path propagation environments, the target is reconstructed at the correct location with correct velocity.

Comparing Fig. 3 with Fig. 2, we observe that the strength of the reconstructed target increases in the multiple-scattering environment, which indicates that the multi-path propagation improves the imaging performance. We also note the additional artifacts present in the background of the reconstructed images.A detailed analysis of the effect of multiple scattering on the resolution of the reconstructed images will be the focus of our future work.

\section{CONCLUSION}

In this work, we presented a new passive imaging method for moving targets in position and velocity spaces using sparsely distributed receivers in multiple-scattering environment. This method is capable of exploiting the multiple scattering to improve reconstructed images. We developed a passive measurement model for moving targets in multiple-scattering environments. We formulated the passive image formation problem within a GLRT framework where we set up a test of binary hypothesis using the passive measurement model for a hypothetical target located at an unknown position, moving with an unknown velocity. We designed a linear discriminant functional by maximizing the SNR of the test-statistic. The resulting discriminant functional correlates the filtered, delayed and scaled (or dilated) averaged measurements from one receiver location with the measurements at another receiver location.

We presented numerical simulation results to demonstrate the performance of our passive imaging method. The resolution analysis of our imaging method will be the focus of our future work.

While we mainly focused on the passive radar application, the results presented in our paper are also applicable to other wave-based passive imaging applications, such as those in acoustic or seismic imaging.

\section{REFERENCES}

[1] Griffiths, H. D. and Long, N. R. W., "Television-based bistatic radar," IEE Proceedings of Radar, Sonar and Navigation 133, 649-657 (July 1986).

[2] Griffiths, H. D. and Baker, C. J., "Passive coherent location radar systems. part 1: Performance prediction," IEE Proceedings of Radar, Sonar and Navigation 152, 153-159 (June 2005).

[3] Baker, C. J., Griffiths, H. D., and Papoutsis, I., "Passive coherent location radar systems. part 2: Waveform properties," IEE Proceedings of Radar, Sonar, and Navigation 152, 160-168 (June 2005).

[4] O'Hagan, D. W. and Baker, C. J., "Passive bistatic radar (PBR) using FM radio illuminators of opportunity," in [Proc. of 2008 IEEE Radar Conference, Roma, Italy], (May 2008).

[5] Koch, V. and Westphal, R., "New approach to a multistatic passive radar sensor for air/space defense," IEEE Aero. Electron. Syst. Mag. 10, 24-32 (Nov. 1995). 


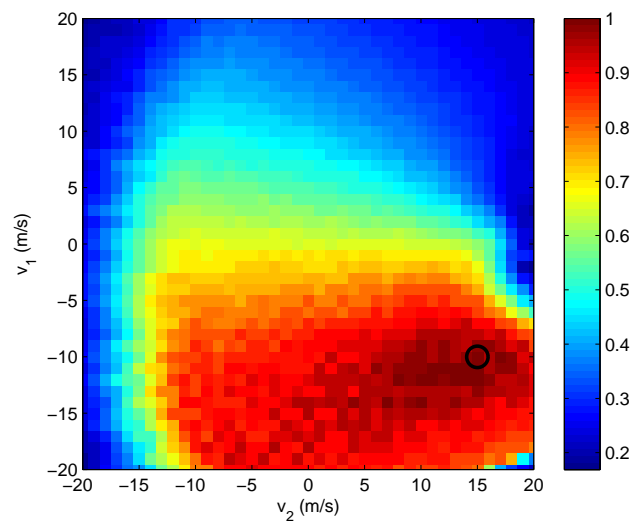

(a)

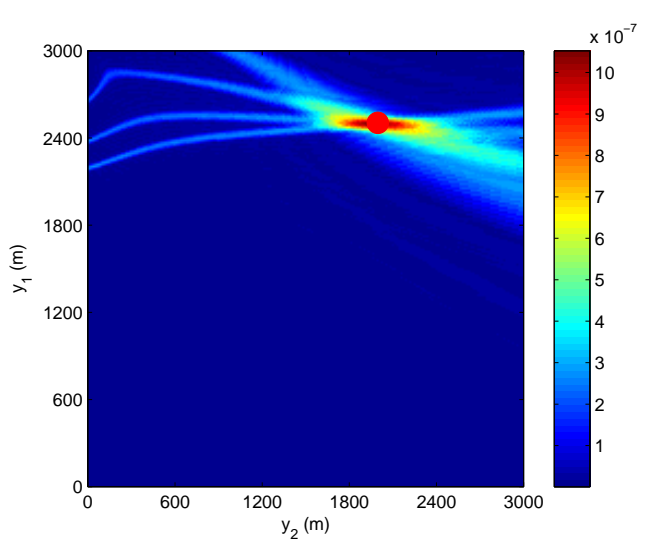

(b)

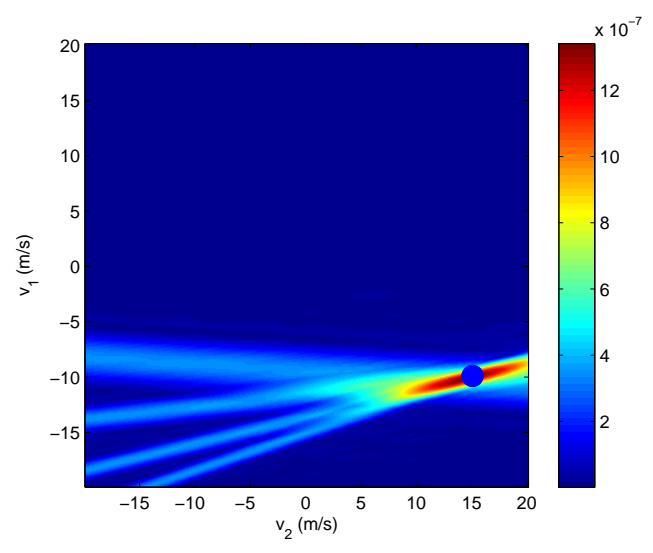

(c)

Figure 2: The reconstructed images in free-space for a moving point target with 5 receivers and a single transmitter transmitting a high-Doppler and good-range resolution waveform: (a) The peak-value image with the maximum value indicated by a circle. The estimated velocity $\tilde{\boldsymbol{v}}=(-10,15) \mathrm{m} / \mathrm{s}$. (b) The position-image when $\boldsymbol{v}=\tilde{\boldsymbol{v}}$. The estimated position $\tilde{\boldsymbol{y}}=(2500,2000) \mathrm{m}$. (c) The velocity-image when $\boldsymbol{y}=\tilde{\boldsymbol{y}}$. Solid dots indicate the true position (or velocity) and circles indicate the estimated position (or velocity)

[6] Poullin, D., "Passive detection using digital broadcasters (DAB, DVB) with COFDM modulation," IEE Proceedings of Radar, Sonar and Navigation 152, 143-152 (June 2005).

[7] Tan, D. K. P., Sun, H., Lu, Y., Lesturgie, M., and Chan, H. L., "Passive radar using global system for mobile communication signal: theory, implementation and measurements," IEE Proceedings of Radar, Sonar, and Navigation 152, 116-123 (June 2005).

[8] Howland, P. E., Maksimiuk, D., and Reitsma, G., "Fm radio based bistatic radar," IEE Proceedings of Radar, Sonar and Navigation 152, 107-115 (June 2005).

[9] He, X., Cherniakov, M., and Zeng, T., "Signal detectability in SS-BSAR with GNSS non-cooperative transmitter," IEE Proceedings of Radar, Sonar and Navigation 152, 124-132 (June 2005).

[10] Kulpa, K. S., "Multi-static entirely passive detection of moving targets and its limitations," IEE Proceedings of Radar, Sonar, and Navigation 152, 169-173 (June 2005).

[11] Coleman, C. and Yardley, H., "Passive bistatic radar based on target illumniations by digital audio broadcasting," IET Radar Sonar Navig. 2(5), 366-375 (2008).

[12] Guo, H., Woodbridge, K., and Baker, C. J., "Evaluation of WiFi beacon transmissions for wireless based passive radar," in [Proc. of 2008 IEEE Radar Conference, Roma, Italy], (May 2008). 


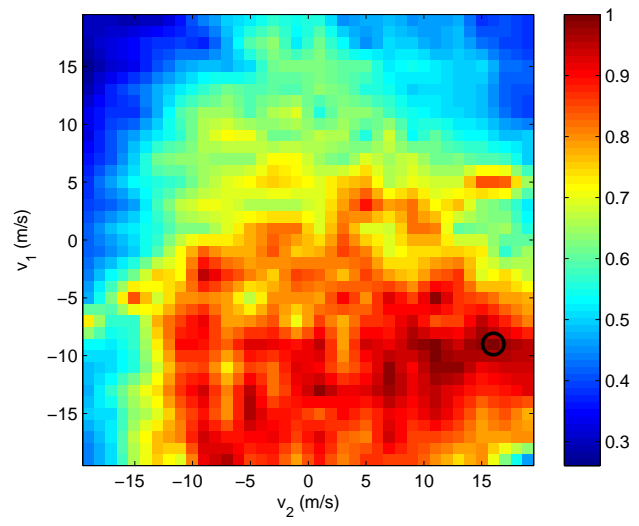

(a)

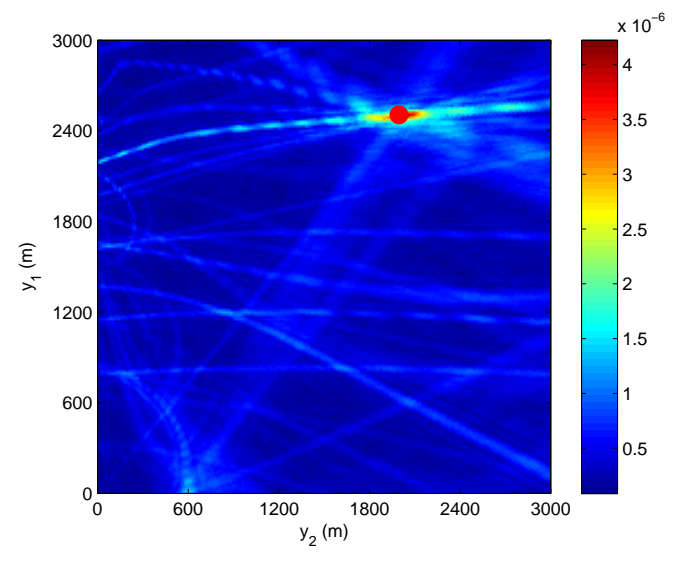

(b)

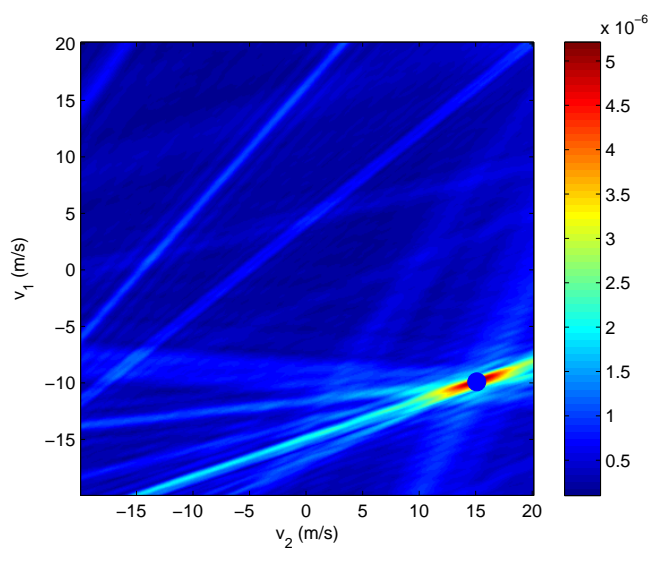

(c)

Figure 3: The reconstructed images in multiple-scattering environment for a moving point target with 5 receivers and a single transmitter transmitting a high-Doppler and good-range resolution waveform: (a) The peak-value image with the maximum value indicated by a circle. The estimated velocity $\tilde{\boldsymbol{v}}=(-10,15) \mathrm{m} / \mathrm{s}$. (b) The position-image when $\boldsymbol{v}=\tilde{\boldsymbol{v}}$. The estimated position $\tilde{\boldsymbol{y}}=(2500,2000) \mathrm{m}$. (c) The velocity-image when $\boldsymbol{y}=\tilde{\boldsymbol{y}}$. Solid dots indicate the true position (or velocity) and circles indicate the estimated position (or velocity)

[13] Chetty, K., Woodbridge, K., Guo, H., and Smith, G. E., "Passive bistatic WiMAX radar for marine surveillance," in [Proc. of 2010 IEEE Radar Conference, Washington, DC, USA], (May 2010).

[14] Falcone, P., Colone, F., Bongioanni, C., and Lombardo, P., "Experimental results for OFDM WiFi-based passive bistatic radar," in [Proc. of 2010 IEEE Radar Conference, Washington, DC, USA], (May 2010).

[15] Harms, H. A., Davis, L. M., and Palmer, J., "Understanding the signal structure in DVB-T signals for passive radar detection," in [Proc. of 2010 IEEE Radar Conference, Washington, DC, USA], (May 2010).

[16] Christiansen, J. M. and Olsen, K. E., "Range and doppler walk in DVB-T based passive bistatic radar," in [Proc. of 2010 IEEE Radar Conference, Washington, DC, USA], (May 2010).

[17] Wu, Y. and Munson, D. C., "Multistatic synthetic aperture imaging of aircraft using reflecting television signals," in [Proc. of SPIE, Algorithms for Synthetic Aperture Radar Imagery VIII], (April 2001).

[18] Wu, Y. and Munson, D. C., "Wide-angle ISAR passive imaging using smoothed pseudo Wigner-Ville distribution," in [Proc. of 2001 IEEE Radar Conference], (May 2001).

[19] Kay, S., [Fundamentals of Statistical Signal Processing, Vol. I - Estimation Theory], Prentice Hall (1993).

[20] Kay, S., [Fundamentals of Statistical Signal Processing, Vol II - Detection Theory], Prentice Hall (1998). 
[21] Colton, D. and Kress, R., [Inverse Acoustic and Electromagnetic Scattering Theory], vol. 93 of Applied Mathematical Sciences, Springer, 2 ed. (1998).

[22] Ghosh Roy, D. N. and Couchman, L. S., [Inverse Problems and Inverse Scattering of Plane Waves], Academic Press, London, UK (2002).

[23] Barrett, H. H. and Myers, K. J., [Foundations of Image Science], Wiley-Interscience (2003).

[24] Cheney, M. and Borden, B., "Imaging moving targets from scattered waves," Inverse Problems 24, 035005(122) (2008).

[25] Cheney, M., "A mathematical tutorial on synthetic aperture radar," SIAM Review 43(2), 301-312 (2001).

[26] Langenberg, K. J., Brandfass, M., Mayer, K., Kreutter, T., Brüll, A., Felinger, P., and Huo, D., "Principles of microwave imaging and inverse scattering," EARSeL Adv. Remote Sens. 2, 163-186 (1993).

[27] Wang, L., Son, I. Y., and Yazıc1, B., "Passive imaging using distributed apertures in multiple scattering environments," Inverse Problems 26, (065002) (2010).

[28] Lax, M., "Multiple scattering of waves," Rev. Mod. Phys 23, 287-310 (1951).

[29] Chew, W. C., [Waves and Fields in Inhomogeneous Media], New York: IEEE Press (1990).

[30] Wang, L. and Yazıc1, B., "Passive imaging of moving targets using sparse distributed apertures," submitted to Inverse Problems.

[31] 802.16e2 005, I. S. and 802.16d2 005, I. S., "Part 16: Air interface for fixed and mobile broadband wireless access systems," 